\title{
Exosomes Derived from Hypoxia-conditioned Adipose-derived Mesenchymal Stem Cells Enhance Lymphangiogenesis via Modulation of miR- 129/HMGB1/AKT Pathway
}

\author{
Yi Yang \\ Sun Yat-sen University First Affiliated Hospital \\ Xu-bo Li \\ Sun Yat-sen University First Affiliated Hospital \\ Yu Li \\ Sun Yat-sen University First Affiliated Hospital \\ Tian-xiao Li
}

Sun Yat-Sen University Guanghua School of Stomatology

\section{Ping Li}

Sun Yat-sen University First Affiliated Hospital

\section{Guang-mao Deng}

Huiya hospital of the first affiliated hospital,Sun Yat-sen

Qiang Guo

Huiya hospital of the first affiliated hospital,Sun Yat-sen

\section{Xiang Zhou}

Sun Yat-sen University First Affiliated Hospital

\section{Xiao-hu Chen ( $D 249004552 @ q q . c o m$ )}

Hui Ya hospital of the first affiliated,sun yet-sen university

\section{Research}

Keywords: Lymphangiogenesis, adipose-derived mesenchymal stem cells, exosomes, AKT, hypoxia

Posted Date: September 8th, 2021

DOI: https://doi.org/10.21203/rs.3.rs-871701/v1

License: () (1) This work is licensed under a Creative Commons Attribution 4.0 International License. Read Full License 


\section{Abstract}

Background Exosomes from adipose-derived mesenchymal stem cells (ADSCs) play an important role in lymphangiogenesis; however, the underlying mechanisms are not fully understood. In this study, we aimed to investigate the function of exosomes secreted by hypoxia-conditioned ADSCs in lymphangiogenesis and explore the potential molecular mechanisms.

Methods Exosomes were extracted from ADSCs cultured under hypoxia or normoxia conditions. The uptake of exosomes by LECs was detected by immunofluorescence staining. The effects of exosomes on the viability, migration, and tube formation of LECs were determined by CCK-8 assay, migration assay, and tube formation assay, respectively. Molecules and pathway involved in lymphangiogenesis mediated by ADSC-derived exosomes were analyzed by luciferase reporter assay, qRT-PCR, and Western blot.

Results Hypoxia ADSC-derived exosomes (H-ADSC/exos) significantly enhanced the proliferation, migration, and tube formation of LECs. Hypoxia decreased the expression of miR-129 in ADSC-derived exosomes. Overexpression of miR-129 counteracted the promoting effect of H-ADSC/exos on lymphangiogenesis. In addition, decreased exosomal miR-129 expression resulted in upregulation of HMGB1 in LECs, which led to AKT activation and lymphangiogenesis enhancement. Our data reveal that exosomes derived from hypoxia-conditioned ADSCs induce lymphangiogenesis, and this effect is mediated by miR-129/HMGB1/AKT signaling.

Conclusions Our findings imply that hypoxia ADSC-isolated exosomes may represent as a valuable target for the treatment of diseases associated with lymphatic remodeling.

\section{Introduction}

Lymphangiogenesis is a process that forms new lymphatic vessels from pre-existing lymphatic vasculature and plays important roles in many diseases, such as cancer, inflammatory bowel disease, and endometriosis [1-3]. Mesenchymal stem cell (MSC)-based therapy has been considered as a promising strategy for lymphatic regeneration [4], but the underlying mechanism is not fully clarified.

Accumulating evidence suggests that MSCs exert their function via secreting vesicles and molecules [5, 6]. Exosomes are cellular vesicles (40-100 $\mathrm{nm}$ in diameter) that are secreted by different types of cells. Exosomes play an critical function in many processes, such as cell proliferation, survival, migration, apoptosis, immune response, by delivering proteins, mRNAs, or miRNAs from host cells to recipient cells $[7,8]$. Adipose-derived mesenchymal stem cells (ADSCs) are a population of pluripotent cells derived from adipose tissue, whose characteristics are similar to bone marrow-derived mesenchymal stem cells (BMSCs) [9]. Multiples studies have reported that ADSCs have potential application for promoting lymphatic generation [10-12]. It has been reported that ADSCs promotes VEGF-C-dependent lymphangiogenesis via secreting exosomes [6], indicating that the paracrine effect is critical for ADSCmediated lymphangiogenesis promotion. 
The oxygen concentration is an important factor for stem cell proliferation and differentiation. In the body, the oxygen content is quite low in adipose tissue and thus ADSCs usually exist in low oxygen conditions [13]. Consistently, treatment with hypoxia can promote the proliferation and differentiation of ADSCs and alter their molecule production [14]. However, the function of exosomes from hypoxiaconditioned ADSCs in lymphangiogenesis remain unclear. In the current study, we investigated the effected of hypoxia ADSC-derived exosomes on lymphatic endothelial cell proliferation, migration and tube formation, and explored the molecules and pathway involved in this process.

\section{Materials And Methods}

\section{Cell culture}

Human ADSCs and dermal lymphatic endothelial cells (LECs) were obtained from Guangzhou Cyagen Biology (Huangpu District, Guangzhou, China). ADSCs were cultured in DMEM medium (Gibco, Waltham, MA, USA) containing 10\% FBS (Gibco, USA) and 1\% penicillin-streptomycin (Gibco, USA) under hypoxia $\left(5 \% \mathrm{O}_{2}\right)$ or normoxia conditions $\left(21 \% \mathrm{O}_{2}\right)$. LECs were maintained in endothelial growth medium-2-MV (EGM-2-MV, Lonza) that contains endothelial basal medium-2 (EBM-2, Lonza) and SingleQuots kit (Lonza).

\section{Exosome isolation}

ADSCs were cultured under hypoxia or normoxia conditions for 6 days and the medium was harvested for exosome isolation. The medium was centrifuged at $3,000 \mathrm{~g}$ for $15 \mathrm{~min}$ and then $20,000 \mathrm{~g}$ for $45 \mathrm{~min}$ at $4^{\circ} \mathrm{C}$. The supernatant was filtered and further centrifuged at $110,000 \mathrm{~g}$ for $70 \mathrm{~min}$ at $4^{\circ} \mathrm{C}$. The sample were washed once with PBS and then recentrifuged at $110,000 \mathrm{~g}$ for another $70 \mathrm{~min}$ at $4^{\circ} \mathrm{C}$.

\section{Transmission electron microscopy}

Exosomes isolated from ADSCs were resuspended in PBS. A drop of exosomes was placed on the top of a copper grid. After washing once with PBS, exosomes were fixed with $3 \%$ glutaraldehyde for $15 \mathrm{~min}$. The sample was washed again with PBS and incubated with $2 \%$ uranyl acetate for $5 \mathrm{~min}$. Then, exosomes were visualized under a transmission electron microscope. Dynamic light scattering was used to determine the exosome size.

\section{Uptake of exosomes}

ADSC-derived exosomes were labelled with KH67 dye (Sigma, USA). LECs were seeded in 6-well plates and $2 \mu \mathrm{g} \mathrm{KH67-stained} \mathrm{exosomes} \mathrm{were} \mathrm{added.} \mathrm{After} \mathrm{incubation} \mathrm{for} 24 \mathrm{~h}$, LECs were fixed with $4 \%$ 
paraformaldehyde. After stained with DAPI, LECs were analyzed under a confocal microscope (Carl Zeiss, Germany), and the location of exosomes was visualized.

\section{CCK-8 assay}

LECs (2,000 cells/well) in $100 \mu$ medium were seeded in 96-well plate. For detecting the viability of LECs, $10 \mu \mathrm{l}$ Cell Counting Kit-8 (CCK-8) reagent (Seyotin, China) was added to each well. After incubation for $1 \sim 4 \mathrm{~h}$, the absorbance at $450 \mathrm{~nm}$ was determined by a plate reader.

\section{Transwell migration assay}

LECs $\left(1 \times 10^{5}\right)$ in $100 \mu$ l serum-free medium were added into Transwell inserts (Corning, USA), and $600 \mu$ l completed medium with $10 \mu \mathrm{g} / \mathrm{ml}$ exosomes was added to the lower compartment of the chamber. After incubation for $24 \mathrm{~h}$, LECs were fixed with methanol for $10 \mathrm{~min}$ and stained with $0.0 .5 \%$ crystal violet for $15 \mathrm{~min}$. The non-migrating cells were scraped with a cotton swab and the migrated cells were visualized under an inverted microscope (Carl Zeiss, Germany).

\section{Tube formation analysis}

Twenty-four-well plates were precoated with Matrigel (BD Sciences, USA) for $30 \mathrm{~min}$ at $37^{\circ} \mathrm{C}$ prior to cell seeding. LECs $(2 \times 104$ cells/well) were seeded in 24-well plates and $10 \mu \mathrm{g} / \mathrm{ml}$ ADSC-derived exosomes were added. After incubation for $24 \mathrm{~h}$, the tube number at three random field was counted using a light microscope.

\section{Quantitative RT-PCR}

LECs were treated with ADSC-derived exosomes $(10 \mu \mathrm{g} / \mathrm{ml})$ for $24 \mathrm{~h}$. LECs were collected and used for RNA isolation. Total RNA was extracted using TRIzol reagent (Life Science, Waltham, MA, USA) and was reverse transcribed to cDNA using the PrimeScript RT-PCR kit (Seyotin, China). qPCR was performed with the qPCR Mix (Seyotin, china) on the Applied Biosystems 7300 system (Applied Biosystems, USA). The experiments were conducted in triplicate. Gene (or miRNA) expression was normalized to GAPDH (or U6) using the $2^{-\triangle \Delta C T}$ formula.

\section{DNA and RNA transfection}

miR-129 mimics and control mimics (NC mimics) were purchased from RiboBio (Guangzhou, China). HMGB1 expression plasmid and control plasmid were obtained from Hanbio (Shanghai, China). miRNA 
mimics and HMGB1 expression plasmid were transfected into LECs using Lipofectamine 2000 (ThermoFisher, USA) according to the manufacturer's instructions.

\section{Luciferase reporter assay}

The wild type and mutated HMGB1 3'UTR were cloned into pGL3 plasmid to form recombinant plasmids pGL3-HMGB1-3'UTR-wt and pGL3-HMGB1-3'UTR-mut. LECs (2×105 cells/well) were seeded in 24-well plates and were cotransfected with pGL3-HMGB1-3'UTR-wt (or pGL3-HMGB1-3'UTR-mut), miR-129 mimics (or NC mimics), and pRL-TK (Promega, USA). After transfection for $48 \mathrm{~h}$, the luciferase activity was determined by using a Dual-Luciferase Reporter Assay System (Promega).

\section{Western blot}

LECs or ADSC-derived exosomes were lysed using RIPA lysis buffer. The sample was separated by SDSPAGE gel and transferred to PVDF membranes (Millipore, USA). After blocking with $5 \%$ non-fat milk, the PVDF membrane was incubated with primary antibody at $4^{\circ} \mathrm{C}$ overnight. After washing three times with TSB-Tween 20 (TBST), the membrane was incubated with the secondary antibody for $1.5 \mathrm{~h}$ at room temperature. After three washes with TBST, the protein bands were detected by using the Western Blot Detection Kit (Seyotin, China). The antibodies used were as follows: CD9 (ab59479; Abcam, UK), CD63 (ab59479; Abcam), TSG-101 (ab125011; Abcam, UK), HMGB1 (ab18256; Abcam, UK), LYVE-1 (ab14917; Abcam, UK), PROX1 (ab101851; Abcam, UK), GAPDH (\#2118; Cell Signaling Technology, USA), AKT (\#9272; Cell Signaling Technology, USA), p-AKT (\#4060; Cell Signaling Technology, USA).

\section{Statistical analysis}

The data were represented as the mean \pm standard deviation (SD) from three independent experiments. Statistical comparisons were performed with Student t-test (two groups) or one-way ANOVA (more than two groups). A P value lower than 0.05 was considered as statistically significant.

\section{Results}

\section{Characterization of exosomes derived from ADSCs}

To identify the potential activity of MSC-derived exosomes on lymphangiogenesis, exosomes were isolated from ADSCs cultured under hypoxia (H-ADSC/exos) and normoxia conditions (N-ADSC/exos). Transmission electron microscopy analysis showed that exosomes were spherical and of 100 150 nm diameter and the morphology and size of $\mathrm{H}-\mathrm{ADSC} / \mathrm{exos}$ and $\mathrm{N}-\mathrm{ADSC} / \mathrm{exos}$ were similar (Fig. A). To further confirm the exosomes, the expression of exosomal markers (CD9, CD63, and TSG-101) were analyzed by Western blot. The expression of CD9, CD63, and TSG-101 was detected in both $\mathrm{H}-\mathrm{ADSC} / \mathrm{exos}$ and $\mathrm{N}$ - 
ADSC/exos (Fig. 2B). Next, the internalization of exosomes by LECs were investigated by immunofluorescence staining. The result showed that PKH67-labelled exosomes could be taken up by LECs (Fig. 1C).

\section{H-ADSC/exos increases LEC proliferation, migration, and tube formation}

To investigate whether $\mathrm{H}-\mathrm{ADSC} / \mathrm{exos}$ could influence lymphangiogenesis, we detected the effects of $\mathrm{H}$ ADSC/exos on the proliferation, migration, and tube formation in LECs. After treatment with H-ADSC/exos and $\mathrm{N}-\mathrm{ADSC} / \mathrm{exos}$, the viability of LECs were analyzed byCCK-8. The result showed that $\mathrm{H}-\mathrm{ADSC} / \mathrm{exos}$ significantly increased the viability of LECs compared with N-ADSC/exos (Fig. 2A). Migration assay also showed that treatment with $\mathrm{H}-\mathrm{ADSC} /$ exo highly elevated the migration activity of LECs compared with $\mathrm{N}$ ADSC/exos (Fig. 2B). In addition, tube number was significantly increased in H-ADSC/exos-treated group compared with the N-ADSC/exos-treated group (Fig. 2C). Moreover, $\mathrm{H}-\mathrm{ADSC} / \mathrm{exos}$ highly increased the mRNA and protein expression of lymphangiogenesis modulators, LYVE-1 and PROX1, compared with NADSC/exo (Fig. 2D, E). Together, these data indicate that H-ADSC/exos has a positive effect on LEC proliferation, migration, and tube formation.

\section{Hypoxia-conditioned ADSC/exos promote lymphangiogenesis by reducing miR-129}

Given the important role of miRNAs in lymphangiogenesis, we tested the expression of several miRNAs in exosomes from ADSCs under hypoxia or normoxia conditions. The result showed that miR-129 was significantly decreased in H-ADSC/exos compared with N-ADSCs, while other miRNAs were not altered obviously (Fig. 3A). To access the contribution of miR-129 to H-ADSC/exos-mediated lymphangiogenesis, we transfected miR-129 mimic to LECs under hypoxia or normoxia conditions before exosome extraction. Cell viability analysis and Transwell migration assay showed that treatment with miR-129 decreased the viability and migration of LECs that were increased by H-ADSC/exos (Fig. 3B and C). Additionally, tube formation assay revealed that $\mathrm{H}-\mathrm{ADSC} /$ exos increased tube formation of LECs, and transfection of miR129 mimics reversed this effect (Fig. 3D). Collectively, these results suggest that H-ADSC/exos enhances lymphangiogenesis via reduction of miR-129.

\section{miR-129 suppresses H-ADSC/exos-mediated lymphangiogenesis via HMGB1}

miRNAs exert their functions usually via modulation of downstream target genes. HMGB1 has been reported to play an important role in lymphangiogenesis and its association with miR-129 has been indicated previously [15]. Our data showed that treatment with H-ADSC/exos increased the expression of 
HMGB1, and this effect was blocked by overexpression of miR-129 (Fig. 4A). Luciferase reporter assay showed that transfection with miR-129 mimics could reduce the luciferase activity of HMGB1 3'UTR, but had no significant effect on HMGB1 3'UTR mutant in LECs (Fig. 4B and C), indicating that HMGB1 is a direct target of miR-129 in LECs. Next, we investigated whether miR-129 inhibited lymphangiogenesis via regulating HMGB1. CCK-8 assay showed that miR-129 suppressed H-ADSC/exos-promoted LEC viability and this effect was reversed by overexpression of HMGB1 (Fig. 4D). Similarly, while miR-129 reduced the migration and tube formation of LECs that were promoted by H-ADSC/exos, ectopic expression of HMGB1 counteracted these effects of miR-129 (Fig. 4E and F). Collectively, these data suggest that $\mathrm{H}$ ADSC/exos promotes lymphangiogenesis via modulating miR-129/HMGB1 cascade.

\section{HMGB1 induces lymphangiogenesis via activation of AKT}

To understand the possible pathway by which HMGB1 induced lymphangiogenesis, we tested the expression and phosphorylation of AKT, which is implicated in lymphangiogenesis. Western blot analysis showed that treatment with $\mathrm{H}-\mathrm{ADSC} / \mathrm{exos}$ markedly increased the phosphorylation of AKT, but had no obvious effect on its protein level (Fig. 5A), indicating that H-ADSC/exos could activate AKT signaling. To confirm the involvement of the AKT pathway in H-ADSC/exos-mediated lymphangiogenesis, LECs were treated with AKT inhibitor perifosine. Transwell migration assay showed that $\mathrm{H}-\mathrm{ADSC} / \mathrm{exos}$ increased LCE migration, and treatment with perifosine restrained this promotion (Fig. 5B). Also, administration of perifosine decreased the tube number which was increased by $\mathrm{H}-\mathrm{ADSC} / \mathrm{exos}$. Taken together, these data indicate that H-ADSC/exos enhances lymphangiogenesis via HMGB1-mediated activation of AKT signaling.

\section{Discussion}

Lymphangiogenesis is associated with many critical biological progresses. Increasing reports suggest that MSC-derived exosomes are potential targets for lymphangiogenesis. However, the underlying mechanisms remain largely unclear. In the present study, we confirmed the promoting effect of exosomes derived from ADSCs under hypoxia conditions on lymphangiogenesis. Importantly, our results revealed that hypoxia ADSC-derived exosomes induced lymphangiogenesis via activation of AKT signaling through miR-129/HMGB1 cascade.

A number of miRNAs have been reported to play an important role in lymphangiogenesis [16]. For example, it has been reported that miR-155 promotes lymphatic generation by activating STAT3/VEGFC signaling [17]. MiR-199a/b-5p suppresses lymphangiogenesis by modulating DDR1 [18]. miR-129 has been reported to play crucial roles in multiple processes, including cell proliferation, migration, apoptosis, and angiogenesis [19-21]. It has been also reported that miR-129 inhibits lymphangiogenesis in nasopharyngeal cancer via targeting ZIC2 [22]. In the present study, we found that the expression of miR129 was downregulated in H-ADSC/exos-treated LECs compared with N-ADSCs/exos-treated LECs.

Moreover, transfection of miR-129 could block the promoting effect of H-ADSC/exos on LEC proliferation, 
migration, and tube formation. Therefore, our data indicate that the decreased miR-129 expression contributes to H-ADSC/exos-induced lymphatic generation.

HMGB1 is a heparin-binding protein that is involved in many biological processes, such as cell growth, survival, apoptosis, migration, immune response, etc [23-26]. Mutiple studies have demonstrated that HMGB1 also has an important role in lymphagiogenesis. For instance, it has been reported that curcumin suppresses the lymphangiogenesis of gastric cancer cells via suppression of HMGB1/VEGF-D signaling [27]. Han et al. reported that HMGB1 enhances inflammation-mediated lymphangiogenesis through TLR4dependent signaling pathway [28]. Li et al. also showed that HMGB1 activates NF-kB to upregulate VEGF$\mathrm{C}$ and thus promotes lymphangiogenesis and lymphatic node metastasis in colon cancer [29]. Consistently, in our study, we found that HMGB1 was a direct target of miR-129 in LECs. Moreover, downregulation of exosomal miR-129 resulted in increased HMGB1 expression in LECs, which consequently led to elevated LEC migration and tube formation.

Accumulating evidence suggests that the AKT-mediated signaling participates in lymphangiogeneis. It has been reported that Qingjie Fuzheng Granule inhibits lymphangiogenesis via the VEGF-C/VEGFR-3dependent AKT pathway in colorectal cancer [30]. Treatment with human cathelicidin antimicrobial peptide LL-37 enhances lymphangiogenesis in lymphatic endothelial cells through the AKT and ERK signaling pathways [31]. Previous studies have been demonstrated that HMGB1 is an upstream regulator of AKT signaling [32,33]. Here, we found that treatment with H-ADSC/exos increased AKT phosphorylation, and this effect was abolished by HMGB1 silencing. Moreover, treatment with AKT inhibitor perifosine blocked the promoting effects of $\mathrm{H}-\mathrm{ADSC} / \mathrm{exos}$ on LEC migration, as well as the expression levels of LYVE-1 and PROX1. Therefore, HMGB1/AKT signaling is linked to the function of $\mathrm{H}-$ ADSC/exos in lymphangiogenesis.

\section{Conclusions}

Our results showed that exosomes secreted by ADSCs cultured under hypoxia conditions have elevating effects on LEC proliferation, migration, and tube formation. Hypoxia reduced the expression of miR-129 in ADSC-derived exosomes, which led to activation of HMGB1/AKT signaling and thus enhance lymphangiogenesis. Therefore, hypoxia ADSC-secreted exosomes may serve as a novel strategy for the therapy of lymphangiogenesis-associated diseases.

\section{Abbreviations}

MSCs mesenchymal stem cells

HMGB1 High mobility group box 1 protein

ADSCs adipose-derived mesenchymal stem cells

H-ADSC/exos Hypoxia ADSC-derived exosomes 


\section{Declarations}

\section{Conflict of interest}

There exists no competing interest among the authors.

\section{Acknowledgements}

Not applicable

\section{Funding}

This study was supported by the Medical Science and Technology Research Fund project of Guangdong Province(A2020447)

\section{Availability of data and materials}

For data availability, please contact the corresponding author

\section{Ethics approval and consent to participate}

NO

\section{Consent for publication}

Agree

\section{Author contributions}

Yi Yang and Xu-bo designed the experiments and performed the main experiments, ,Yu Li,Tian-xiao Li,Ping Li,Guang-mao Deng,Qiang Guo,Xiang Zhou analyzed the data,Xiao-hu Chen wrote the manuscript and revised it critically for important intellectual content.

\section{References}

1. Hattori K, Ito Y, Honda M, Sekiguchi K, Hosono K, Shibuya M, Unno N, Majima M. Lymphangiogenesis induced by vascular endothelial growth factor receptor 1 signaling contributes to the progression of endometriosis in mice. J Pharmacol Sci. 2020;143:255-63. 
2. Hosono K, Kojo K, Narumiya S, Majima M, Ito Y. Prostaglandin E receptor EP4 stimulates lymphangiogenesis to promote mucosal healing during DSS-induced colitis. Biomed Pharmacother. 2020;128:110264.

3. Hu A, Yang LY, Liang J, Lu D, Zhang JL, Cao FF, Fu JY, Dai WJ, Zhang JF. SIRT2 modulates VEGFDassociated lymphangiogenesis by deacetylating EPAS1 in human head and neck cancer. Mol Carcinog. 2020;59:1280-91.

4. Lee CY, Kang JY, Lim S, Ham O, Chang W, Jang DH. Hypoxic conditioned medium from mesenchymal stem cells promotes lymphangiogenesis by regulation of mitochondrial-related proteins. Stem Cell Res Ther. 2016;7:38.

5. Robering JW, Weigand A, Pfuhlmann R, Horch RE, Beier JP, Boos AM. Mesenchymal stem cells promote lymphangiogenic properties of lymphatic endothelial cells. J Cell Mol Med. 2018.

6. Wang X, Wang H, Cao J, Ye C. Exosomes from Adipose-Derived Stem Cells Promotes VEGF-CDependent Lymphangiogenesis by Regulating miRNA-132/TGF-beta Pathway. Cell Physiol Biochem. 2018;49:160-71.

7. Wortzel I, Dror S, Kenific CM, Lyden D. Exosome-Mediated Metastasis: Communication from a Distance. Dev Cell. 2019;49:347-60.

8. He C, Zheng S, Luo Y, Wang B. Exosome Theranostics: Biology and Translational Medicine. Theranostics. 2018;8:237-55.

9. Kim WS, Park BS, Sung JH, Yang JM, Park SB, Kwak SJ, Park JS. Wound healing effect of adiposederived stem cells: a critical role of secretory factors on human dermal fibroblasts. J Dermatol Sci. 2007;48:15-24.

10. Ahmadzadeh N, Robering JW, Kengelbach-Weigand A, Al-Abboodi M, Beier JP, Horch RE, Boos AM. Human adipose-derived stem cells support lymphangiogenesis in vitro by secretion of lymphangiogenic factors. Exp Cell Res. 2020;388:111816.

11. Ogino R, Hayashida K, Yamakawa S, Morita E. Adipose-Derived Stem Cells Promote Intussusceptive Lymphangiogenesis by Restricting Dermal Fibrosis in Irradiated Tissue of Mice. Int J Mol Sci. 2020; 21.

12. Takeda K, Sowa Y, Nishino K, Itoh K, Fushiki S. Adipose-derived stem cells promote proliferation, migration, and tube formation of lymphatic endothelial cells in vitro by secreting lymphangiogenic factors. Ann Plast Surg. 2015;74:728-36.

13. Rochette L, Mazini L, Malka G, Zeller M, Cottin Y, Vergely C. The Crosstalk of Adipose-Derived Stem Cells (ADSC), Oxidative Stress, and Inflammation in Protective and Adaptive Responses. Int J Mol Sci. 2020; 21.

14. Hwang OK, Noh YW, Hong JT, Lee JW. Hypoxia Pretreatment Promotes Chondrocyte Differentiation of Human Adipose-Derived Stem Cells via Vascular Endothelial Growth Factor. Tissue Eng Regen Med. 2020;17:335-50.

15. Qiu M, Liu D, Fu Q. MiR-129-5p shuttled by human synovial mesenchymal stem cell-derived exosomes relieves IL-1 beta induced osteoarthritis via targeting HMGB1. Life Sci. 2021;269:118987. 
16. Jung HM, Hu CT, Fister AM, Davis AE, Castranova D, Pham VN, Price LM, Weinstein BM. MicroRNAmediated control of developmental lymphangiogenesis. Elife. 2019; 8.

17. Chang Y, Cui M, Fu X, Zhang L, Li X, Li L, Wu J, Sun Z, Zhang X, Li Z, Nan F, Yan J, Zhang M. MiRNA155 regulates lymphangiogenesis in natural killer/T-cell lymphoma by targeting BRG1. Cancer Biol Ther. 2019;20:31-41.

18. Oh S, Seo M, Choi JS, Joo CK, Lee SK. MiR-199a/b-5p Inhibits Lymphangiogenesis by Targeting Discoidin Domain Receptor 1 in Corneal Injury. Mol Cells. 2018;41:93-102.

19. Wan P, Bai X, Yang C, He T, Luo L, Wang Y, Fan M, Wang Z, Lu L, Yin Y, Li S, Guo Q, Song Z. miR-129$5 p$ inhibits proliferation, migration, and invasion in rectal adenocarcinoma cells through targeting E2F7. J Cell Physiol. 2020;235:5689-701.

20. Li W, Ren Y, Meng T, Yang W, Zhang W. miR-129-5p attenuates hypoxia-induced apoptosis in rat H9c2 cardiomyocytes by activating autophagy. J Gene Med. 2020;22:e3200.

21. Zeng A, Yin J, Li Y, Li R, Wang Z, Zhou X, Jin X, Shen F, Yan W, You Y. miR-129-5p targets Wnt5a to block PKC/ERK/NF-kappaB and JNK pathways in glioblastoma. Cell Death Dis. 2018;9:394.

22. Yu D, Han GH, Zhao X, Liu X, Xue K, Wang D, Xu CB. MicroRNA-129-5p suppresses nasopharyngeal carcinoma lymphangiogenesis and lymph node metastasis by targeting ZIC2. Cell Oncol (Dordr). 2020;43:249-61.

23. Rouhiainen A, Kuja-Panula J, Wilkman E, Pakkanen J, Stenfors J, Tuominen RK, Lepantalo M, Carpen O, Parkkinen J, Rauvala H. Regulation of monocyte migration by amphoterin (HMGB1). Blood. 2004;104:1174-82.

24. Kuniyasu H, Yano S, Sasaki T, Sasahira T, Sone S, Ohmori H. Colon cancer cell-derived high mobility group 1/amphoterin induces growth inhibition and apoptosis in macrophages. Am J Pathol. 2005;166:751-60.

25. Chung H, Nam H, Nguyen-Phuong T, Jang J, Hong SJ, Choi SW, Park SB, Park CG. The blockade of cytoplasmic HMGB1 modulates the autophagy/apoptosis checkpoint in stressed islet beta cells. Biochem Biophys Res Commun. 2021;534:1053-8.

26. Zhang Y, Ren H, Li J, Xue R, Liu H, Zhu Z, Pan C, Lin Y, Hu A, Gou P, Cai J, Zhou J, Zhu W, Shi X. Elevated HMGB1 expression induced by hepatitis $B$ virus $X$ protein promotes epithelial-mesenchymal transition and angiogenesis through STAT3/miR-34a/NF-kappaB in primary liver cancer. Am J Cancer Res. 2021;11:479-94.

27. Da W, Zhang J, Zhang R, Zhu J. Curcumin inhibits the lymphangiogenesis of gastric cancer cells by inhibiton of HMGB1/VEGF-D signaling. Int J Immunopathol Pharmacol. 2019;33:2058738419861600.

28. Han L, Zhang M, Wang M, Jia J, Zhao M, Fan Y, Li X. High Mobility Group Box-1 Promotes Inflammation-Induced Lymphangiogenesis via Toll-Like Receptor 4-Dependent Signalling Pathway. PLoS One. 2016;11:e0154187.

29. Li Y, He J, Zhong D, Li J, Liang H. High-mobility group box 1 protein activating nuclear factor-kappaB to upregulate vascular endothelial growth factor $\mathrm{C}$ is involved in lymphangiogenesis and lymphatic 
node metastasis in colon cancer. J Int Med Res. 2015;43:494-505.

30. Huang B, Lu Y, Gui M, Guan J, Lin M, Zhao J, Mao Q, Lin J. Qingjie Fuzheng Granule suppresses lymphangiogenesis in colorectal cancer via the VEGF-C/VEGFR-3 dependent PI3K/AKT pathway. Biomed Pharmacother. 2021;137:111331.

31. Yanagisawa T, Ishii M, Takahashi M, Fujishima K, Nishimura M. Human cathelicidin antimicrobial peptide LL-37 promotes lymphangiogenesis in lymphatic endothelial cells through the ERK and Akt signaling pathways. Mol Biol Rep. 2020;47:6841-54.

32. Meng L, Li L, Lu S, Li K, Su Z, Wang Y, Fan X, Li X, Zhao G. The protective effect of dexmedetomidine on LPS-induced acute lung injury through the HMGB1-mediated TLR4/NF-kappaB and PI3K/Akt/mTOR pathways. Mol Immunol. 2018;94:7-17.

33. Lv X, Zhu Y, Deng Y, Zhang S, Zhang Q, Zhao B, Li G. Glycyrrhizin improved autophagy flux via HMGB1-dependent Akt/mTOR signaling pathway to prevent Doxorubicin-induced cardiotoxicity. Toxicology. 2020;441:152508.

\section{Figures}


A

N-ADSC/exos

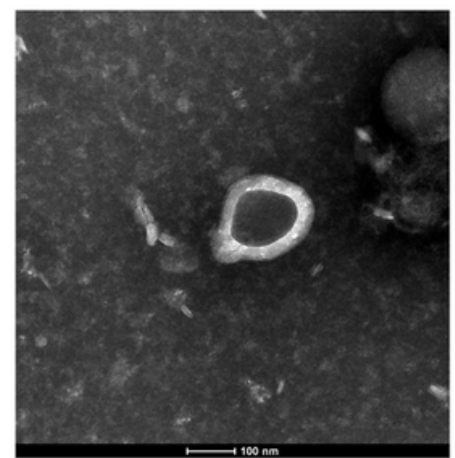

H-ADSC/exos

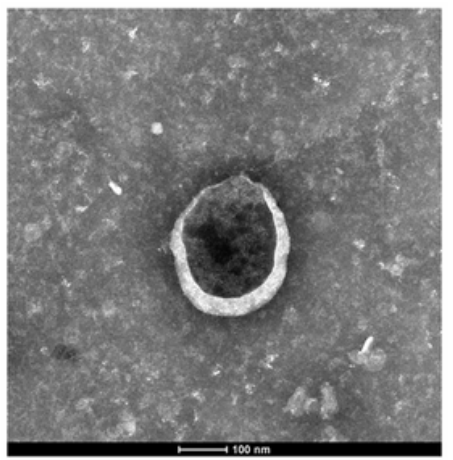

B

\section{N-ADSC/exos H-ADSC/exos}

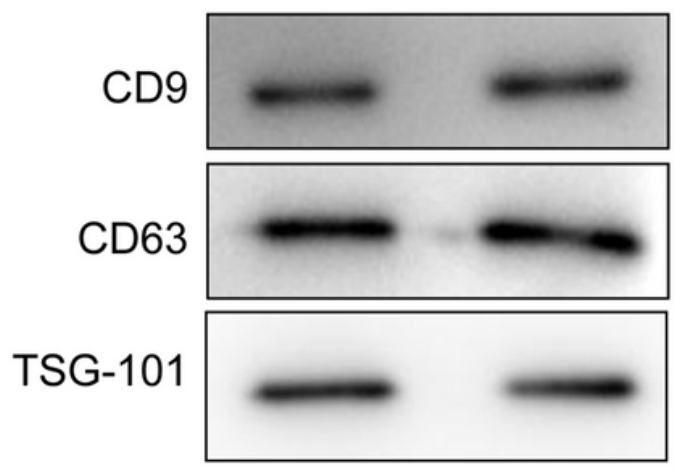

C

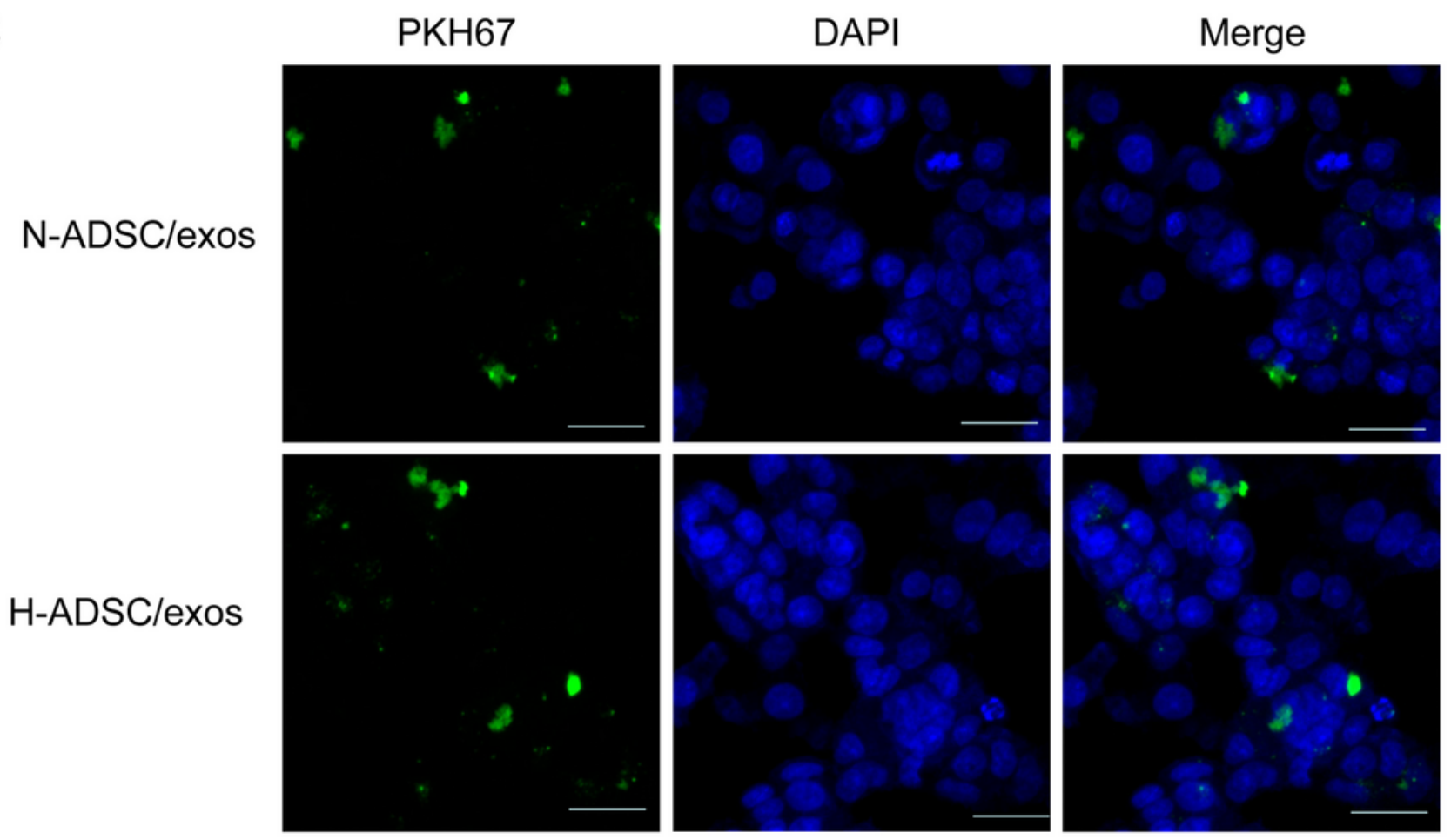

Figure 1

Identification of hypoxia ADSC-derived exosomes. (A). Transmission electron microscopy analysis of the morphology of H-ADSC/exos and N-ADSC/exos. Scale bars: $100 \mathrm{~nm}$. (B) Western blot analysis of CD9, CD63, and TSG-101 expression in of H-ADSC/exos and N-ADSC/exos. (C). Immunofluorescence staining determining the uptake of hypoxia ADSC-derived exosomes by LECs. Data represent the mean \pm SD of three separate experiments; comparison was performed with Student's t-test.Scale bar: $100 \mu \mathrm{m}$. 
A

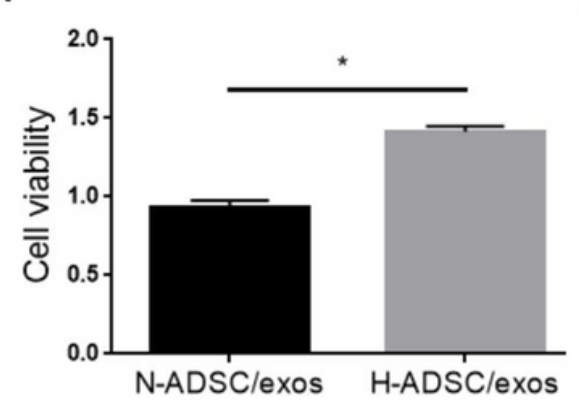

C

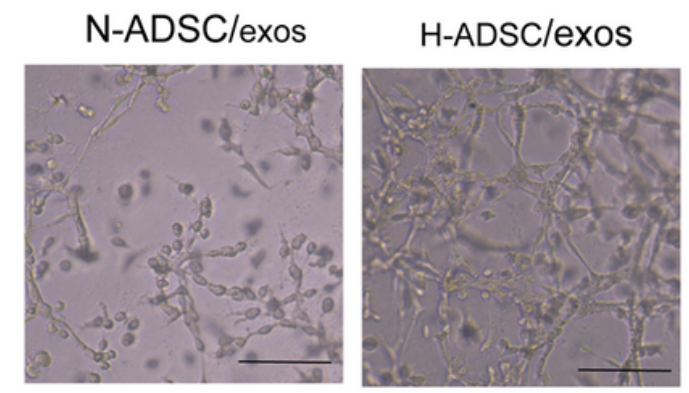

E

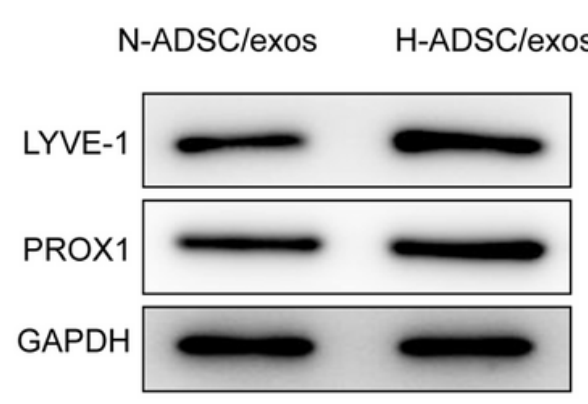

B
N-ADSC/exos
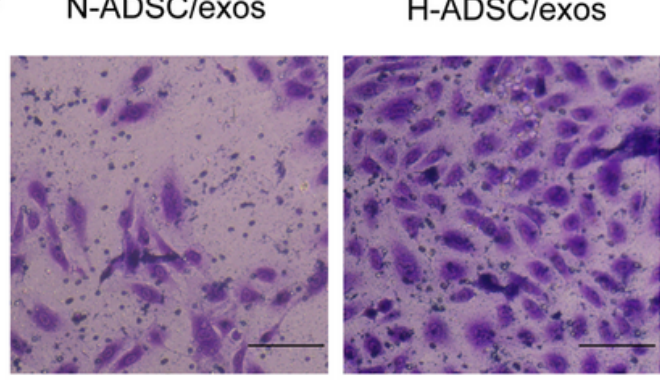

D
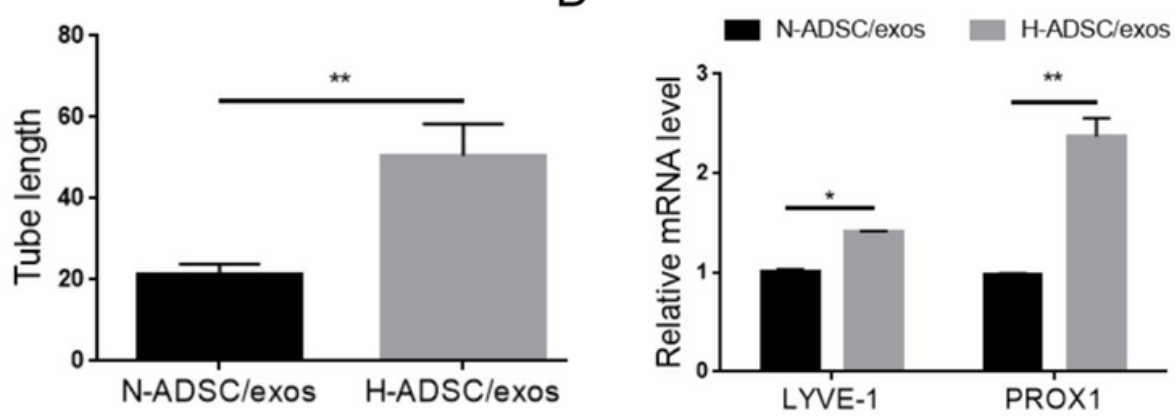

\section{Figure 2}

Effects of hypoxia ADSC-derived exosomes on LEC proliferation, migration, and tube formation. (A) CCK8 assay in LECs after treatment with H-ADSC/exos or N-ADSC/exos. (B) Migration assay in LECs after treatment with $\mathrm{H}-\mathrm{ADSC} /$ exos or N-ADSC/exos. (C) Tube formation assay in LECs after treatment with $\mathrm{H}$ ADSC/exos or N-ADSC/exos. (D, E) qRT-PCR and Western blot analysis of LYVE-1 and PROX1 expression in LECs after treatment with H-ADSC/exos or N-ADSC/exos. ${ }^{*} \mathrm{P}<0.05,{ }^{\star *} \mathrm{P}<0.01$. Data represent the mean \pm SD of three separate experiments; comparison was performed with Student's t-test.Scale bar: 100 $\mu \mathrm{m}$. 

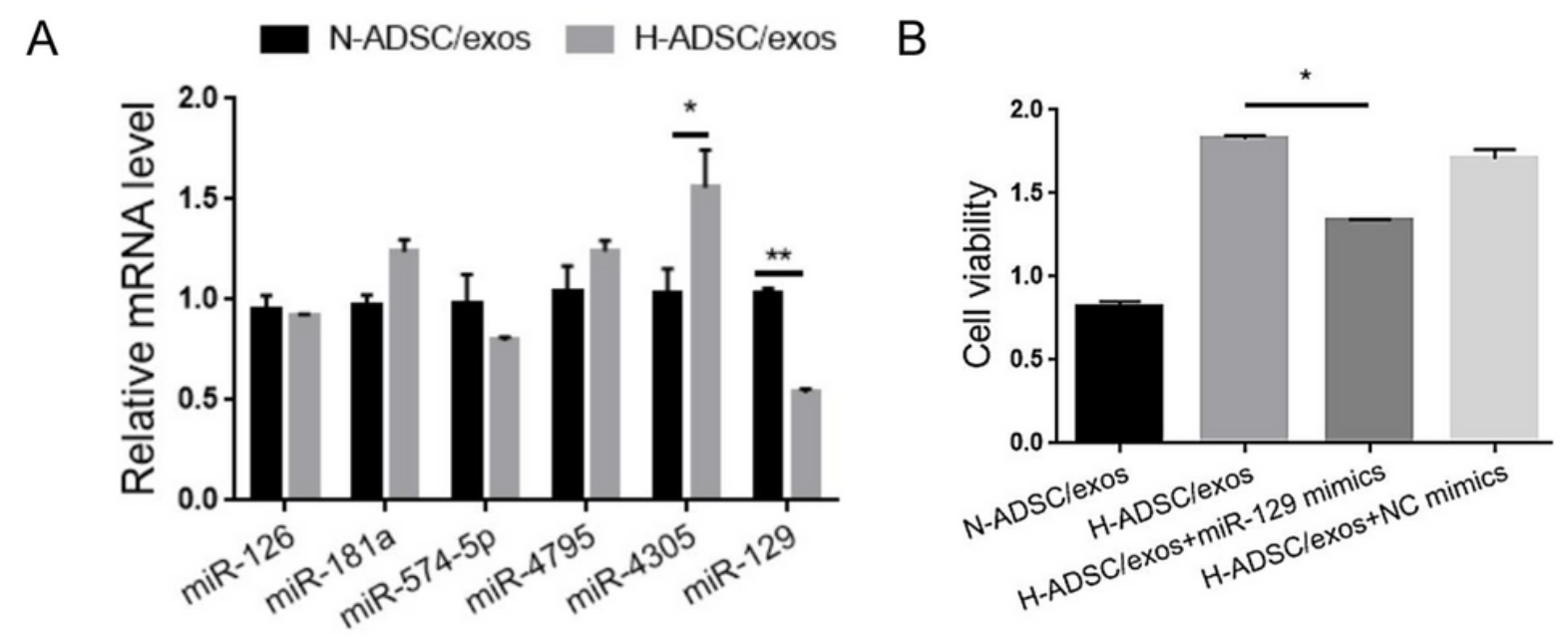

C
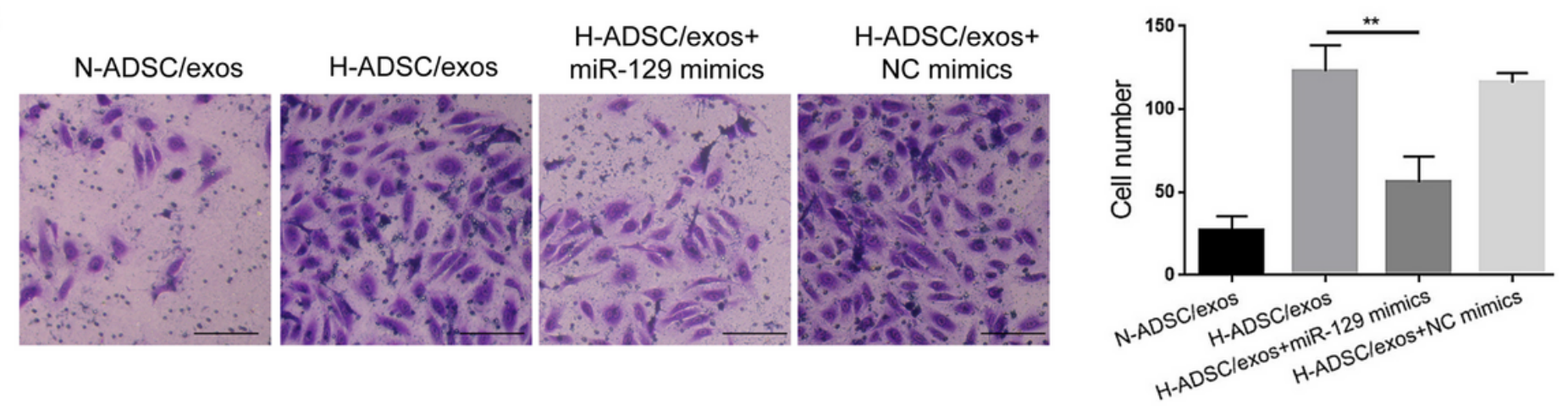

D
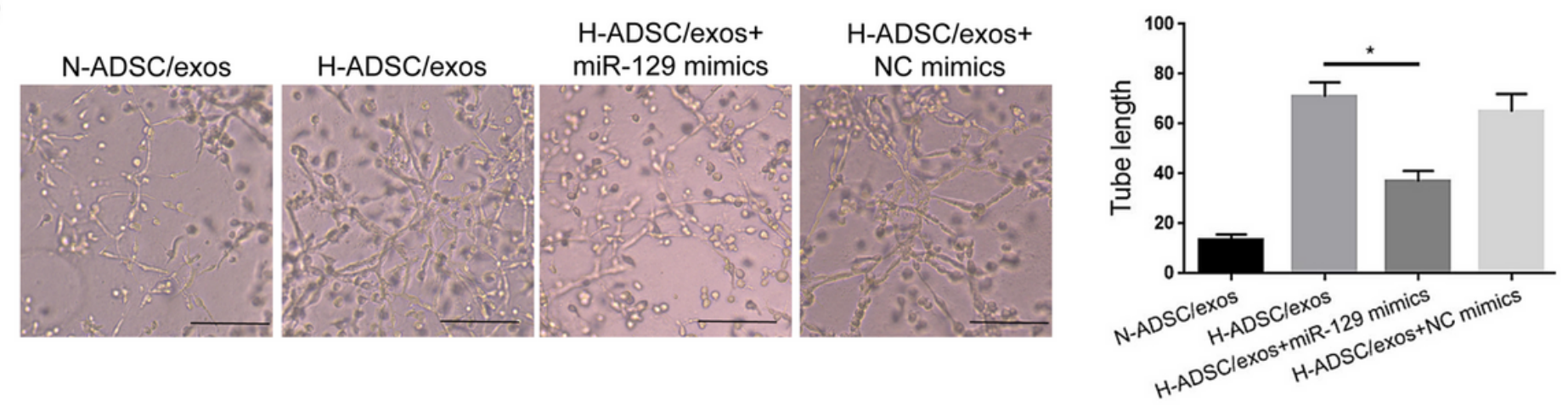

\section{Figure 3}

$\mathrm{H}-\mathrm{ADSC} / \mathrm{exos}$ increase lymphangiogenesis by downregulation of miR-129. (A) qRT-PCR analysis of miRNA expression in LECs treated with H-ADSC/exos or N-ADSC/exos. (B) CCK-8 assay in LECs after treatment with $\mathrm{H}-\mathrm{ADSC} / \mathrm{exos}$ (or N-ADSC/exos) together with miR-129 mimics (or NC mimics). (C) Transwell migration assay in LECs after treatment with H-ADSC/exos (or N-ADSC/exos) together with miR-129 mimics (or NC mimics). (D) Tube formation assay in LECs after treatment with H-ADSC/exos (or $\mathrm{N}-\mathrm{ADSC} / \mathrm{exos}$ ) together with miR-129 mimics (or NC mimics). ${ }^{*} \mathrm{P}<0.05, * \star \mathrm{P}<0.01$. Data represent the mean \pm SD of three separate experiments; comparison was performed with Student's t-test.Scale bar: 100 $\mu \mathrm{m}$. 


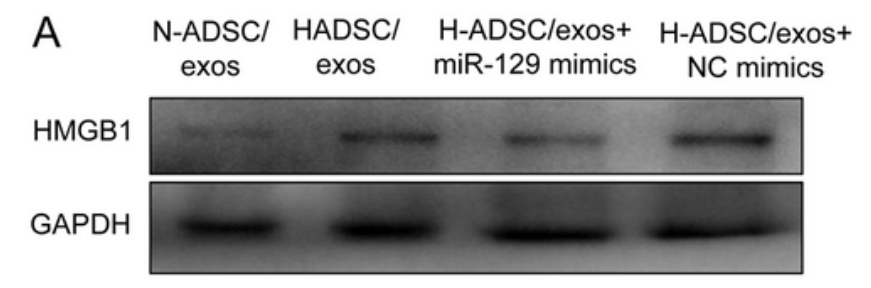

C

E
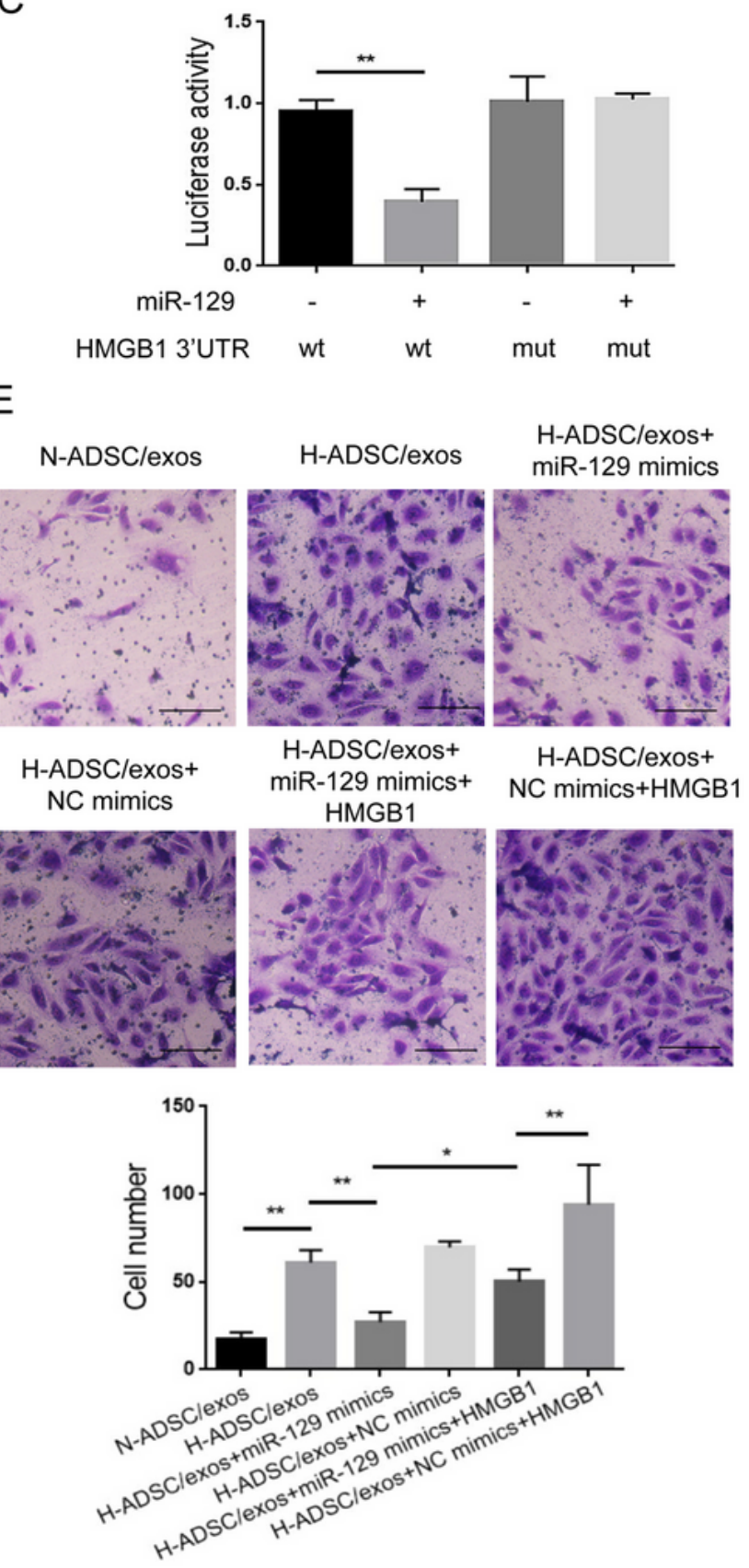

B

hsa-miR-129-5p 3'-CGUUCGGGUCUGGCGUUUUUC-5

HMGB1 3'UTR Wt 5'ACCACUCUGUAAUUGCAAAAAA-3'

HMGB1 3'UTR MU 5'ACCACUCUGUAAUUCGUUUUUA-3'
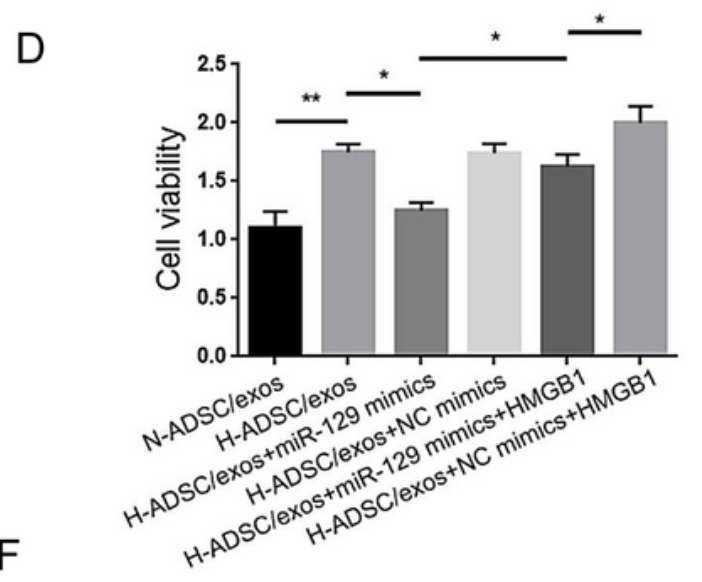

$\mathrm{F}$
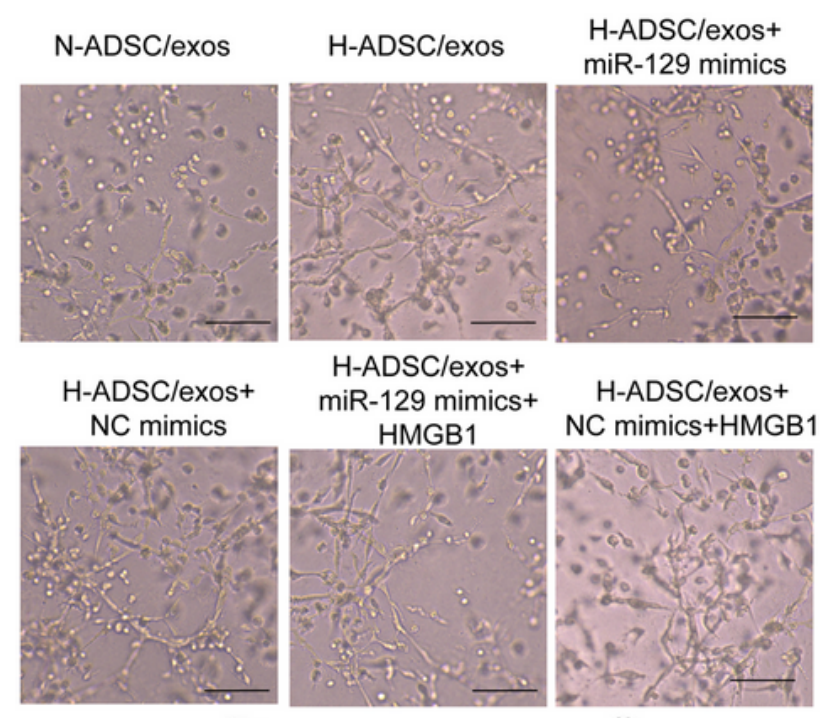

H-ADSClexos+
miR-129 mimics+

H-ADSClexos+ HMGB1 NC mimics+HMGB1

\section{Figure 4}

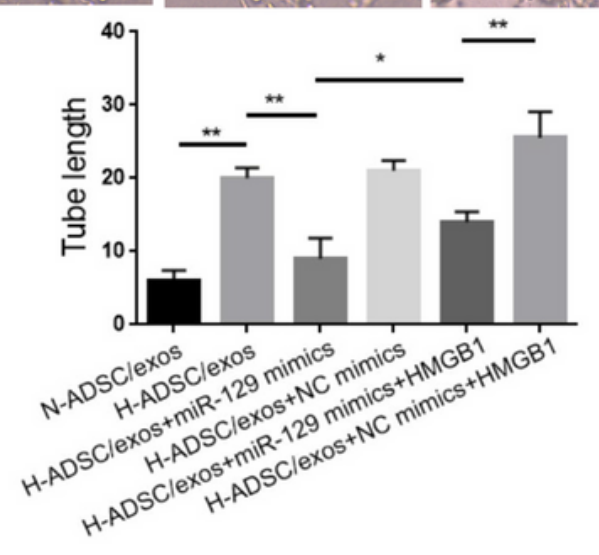

Exosomal miR-129 regulates HMGB1 expression. (A) Western blot analysis of HMGB1 expression in LECs after treatment with H-ADSC/exos with miR-129 mimics or NC mimics. (B) The binding site of miR-129 in HMGB1 3'UTR. (C) Luciferase reporter assay in LECs transfected with miR-129 and wild type (wt) or mutated (mut) HMGB1 3'UTR. (D) CCK-8 assay demonstrated that transfection of miR-129 abolished the effect of H-ADSC/exos on LEC viability, and this effect was reversed by overexpression of HMGB1. (E) 
Transwell migration assay revealed that transfection of miR-129 mimics counteracted the effect of $\mathrm{H}$ ADSC/exos on LEC migration, and this effect was reversed by overexpression of HMGB1. (F) Tube formation assay showed that overexpression of miR-129 restrained the effect of H-ADSC/exos on LEC tube formation, and this effect was reversed by transfection of HMGB1. ${ }^{*}<0.05$, $* * P<0.01$. Data represent the mean \pm SD of three separate experiments; comparison was performed with Student's ttest.Scale bar: $100 \mu \mathrm{m}$.
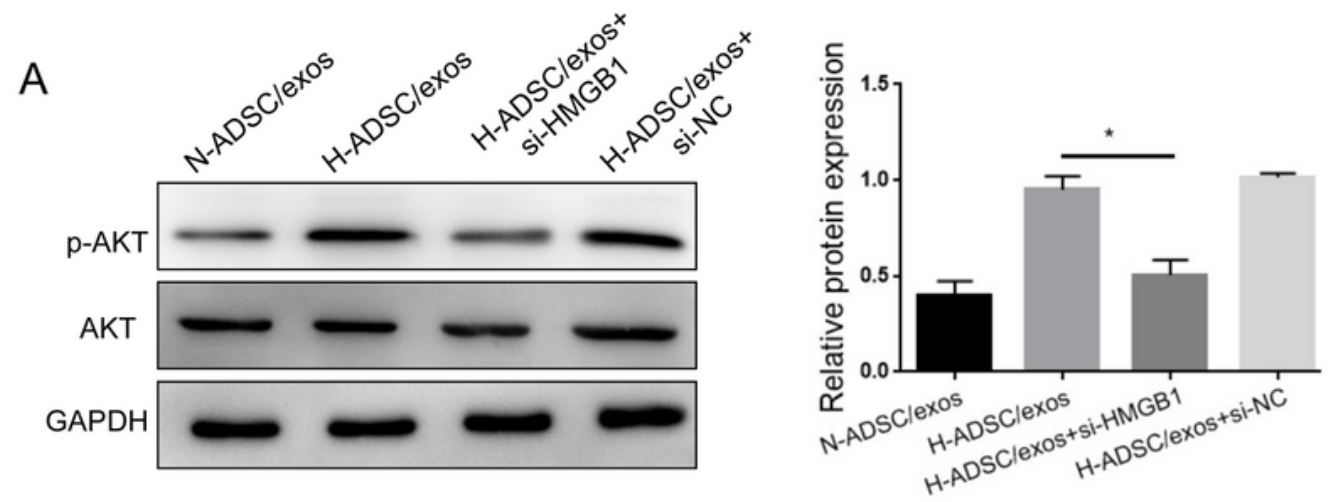

B

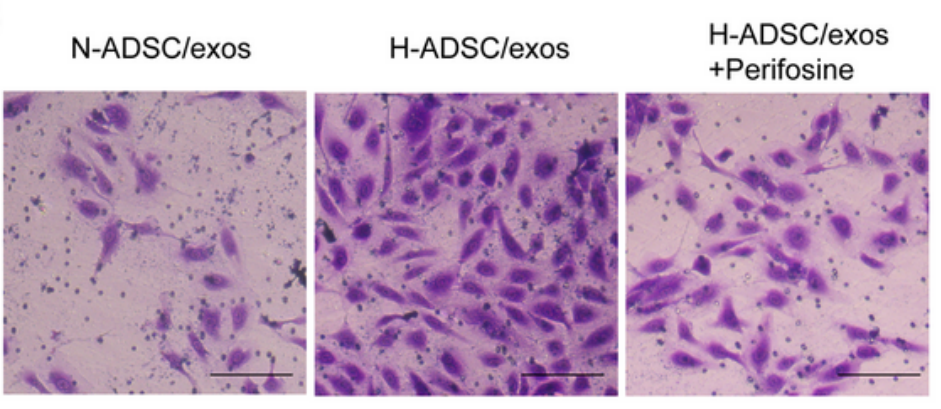

C
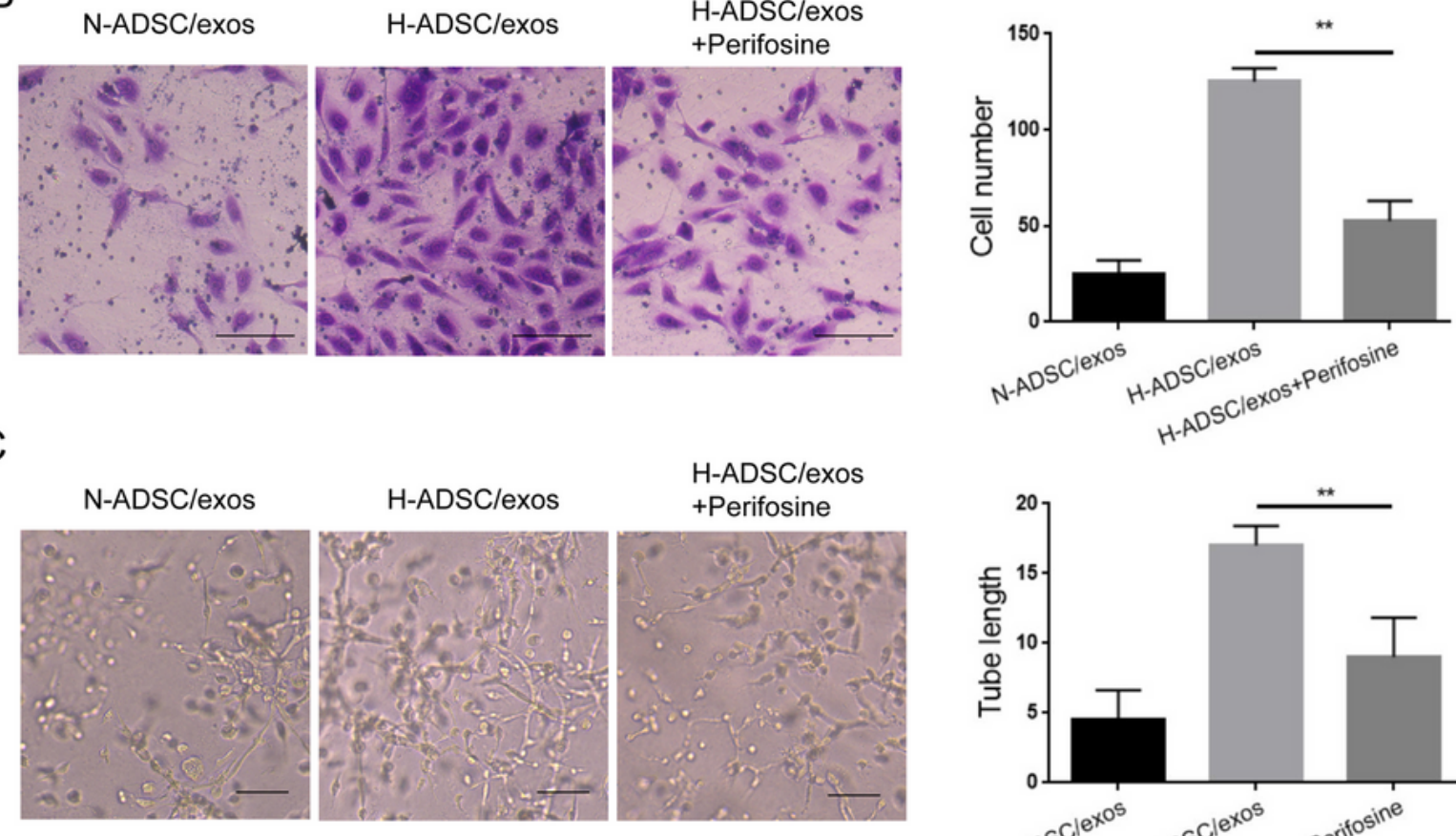

$\mathrm{D}$
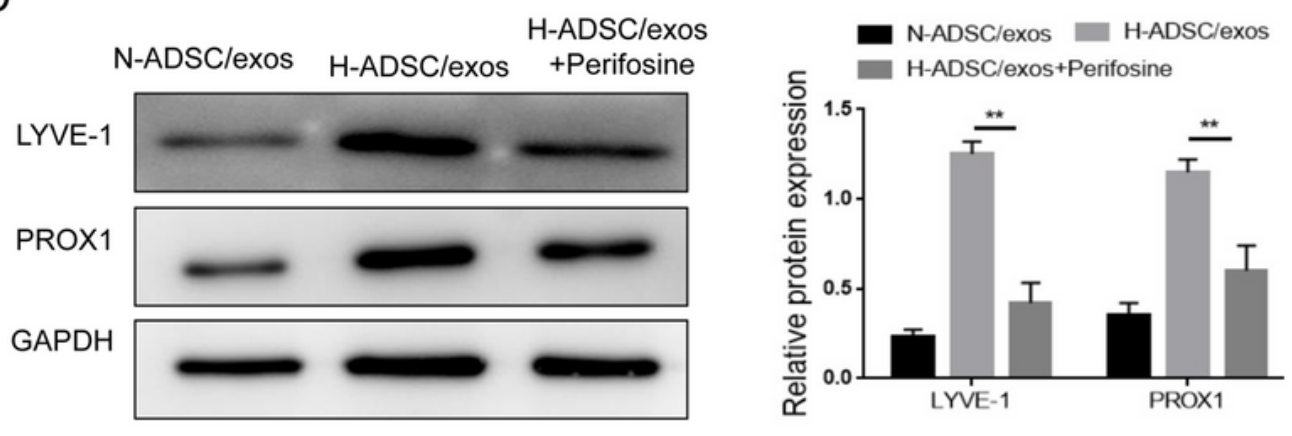

Figure 5 
Hypoxia-conditioned ADSC-derived exosomes activates AKT via HMGB1. (A) Western blot analysis of AKT expression and phosphorylation in LECs after treatment with $\mathrm{H}-\mathrm{ADSC} / \mathrm{exos}$ and transfection with HMGB1 siRNA (si-HMGB1) or control siRNA (si-NC). (B) Treatment with perifosine (30 $\mu \mathrm{M})$ decreased the tube formation of LECs enhanced by H-ADSC/exos. (D) Treatment with perifosine $(30 \mu \mathrm{M})$ reduced $\mathrm{H}$ ADSC/exos-induced LYVE-1 and PROX1 expression. ${ }^{*} P<0.05,{ }^{*} \mathrm{P}<0.01$. Data represent the mean $\pm \mathrm{SD}$ of three separate experiments; comparison was performed with Student's t-test.Scale bar: $100 \mu \mathrm{m}$. 